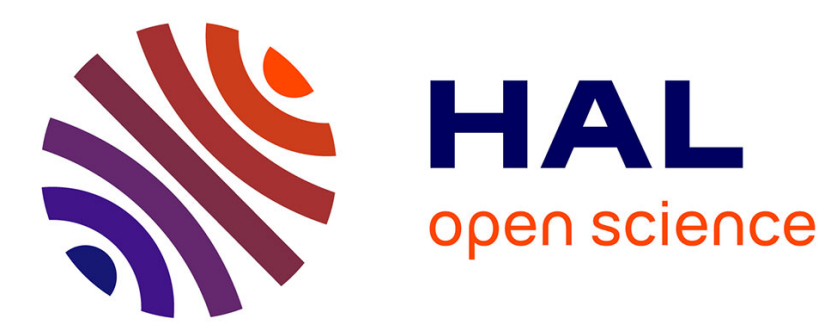

\title{
Dynamics and Thermodynamics of Artificial Muscles Based on Nematic Gels
}

\author{
M. Hébert, R. Kant, P.-G. de Gennes
}

\section{To cite this version:}

M. Hébert, R. Kant, P.-G. de Gennes. Dynamics and Thermodynamics of Artificial Muscles Based on Nematic Gels. Journal de Physique I, 1997, 7 (7), pp.909-919. 10.1051/jp1:1997209 . jpa-00247373

\section{HAL Id: jpa-00247373 https://hal.science/jpa-00247373}

Submitted on 1 Jan 1997

HAL is a multi-disciplinary open access archive for the deposit and dissemination of scientific research documents, whether they are published or not. The documents may come from teaching and research institutions in France or abroad, or from public or private research centers.
L'archive ouverte pluridisciplinaire HAL, est destinée au dépôt et à la diffusion de documents scientifiques de niveau recherche, publiés ou non, émanant des établissements d'enseignement et de recherche français ou étrangers, des laboratoires publics ou privés. 


\title{
Dynamics and Thermodynamics of Artificial Muscles Based on Nematic Gels
}

\author{
M. Hébert (*), R. Kant and P.-G. de Gennes \\ Laboratoire de Physique de la Matière Condensée $\left({ }^{* *}\right)$, Collège de France, \\ 11 Place Marcelin-Berthelot, 75231 Paris Cedex 05, France
}

(Received 2 December 1996, revised 11 March 1997, accepted 13 March 1997)

\author{
PACS.83.10.Nn - Polymer dynamics \\ PACS.87.10.+e - General, theoretical and mathematical biophysics \\ PACS.87.22.Jb - Muscle contraction
}

\begin{abstract}
A scheme based on nemato-mechanical conversion has been proposed for potential artificial muscle applications (de Gennes P.-G., Hébert M. and Kant R., to appear in Macromol. Symp. (1996)). As the temperature in a nematic gel is reduced through the transition temperature, strong uniaxial deformation is encountered. We briefly expose the dynamics of contraction/elongation in this system. Work and dissipative losses are calculated for an operating cycle to get an approximative expression of the ratio work/losses, which can then be compared with real muscular efficiencies.
\end{abstract}

\section{Introduction}

Muscles are irritable tissues: a group of muscular fibers contracts in response to external stimulation. Typical reaction times are in the milliseconds range. During muscular contraction, chemical energy is converted to mechanical work and heat. The muscular efficiency, defined as the ratio of work performed over heat generated during contraction and elongation, runs around $25 \%-30 \%[1]$.

Artificial muscles face the task of reproducing the two main characteristics of real muscle fibers, namely, elasticity and contractility. Two principal schemes have been considered. The first is traditionally based on isotropic swelling of polymeric gels [2,3]. Recently [4], a second one has been proposed; it uses the fact that dry nematic gels (Liquid Single Crystal Elastomers, LSCE [5]) exhibit strong uniaxial deformations upon entering the nematic phase [6].

In both schemes, the elasticity of the fiber is insured by the presence of a polymeric backbone. The difference between them comes from the contractile mechanism. The former possesses both attractive and repulsive interactions between parts of the gel. An external agent (temperature, solvent composition, $\mathrm{pH}$, etc.) tilts the balance between them, thus, driving the system to a sharp phase transition between collapsed and swollen states [7]. The later uses the anisotropy

(*) Author for correspondence (e-mail: Matthieu.Hebert@nortel.ca)

Present address: Speech Research, Dept. 6X14, Nortel Technology, 16 place du Commerce, Ile des Soeurs, Verdun, Québec, H3E-1H6, Canada

$\left({ }^{* *}\right)$ URA-CNRS n ${ }^{\circ} 792$ 
created by the establishment of nematic order, and subsequent deformation of the fiber, as the driving mechanism [4]. The principal defect of the isotropic swelling scheme is that solvent must reach each point of the gel: this process is limited by mass diffusion. This problem is partly circumvented by the dry nematic gel scheme since no solvent is involved. The process is now limited by thermal diffusion $\left(\sim 10^{2}\right.$ times faster than mass diffusion) and must still be improved to be applicable (by bulk heating by a laser or by more complex structures [8], for example).

The main goals of this article are a) to derive the linearized dynamical equations for a nematic gel using a simple phenomenological model, and b) to calculate relevant thermodynamical quantities for a typical contraction/elongation cycle of an artificial muscle. To do so, we will use a canonic form of the dissipation function (quadratic in the time derivatives of the thermodynamic state variables [9]) and a simple symmetry derived form of the free energy (quadratic in the thermodynamical state variables [6]). The approach presented here is valid in the immediate vicinity of the nematic-isotropic transition and, thus, will be unable to account for any non-linear effects, especially soft deformations occurring deep in the nematic phase [10].

The body of this article is as follows. The next Section elaborates on the main steps and conclusions of a prior calculation [4] that was centered on the dynamics of dry nematic gels. The irreversible thermodynamics of the artificial muscle based on nematic gels is developed in Section 3. The aim is to calculate approximately the theoretical muscular efficiency $\eta_{\text {muscular }}=\mid$ work $/$ losses $\mid$.

\section{Dynamics of Dry Nematic Gels [4]}

Let us recall briefly the phenomenological analysis of the continuum dynamics of dry nematic gels and the specific application to thermodynamics of artificial muscles [4]. Special attention is given to the order of magnitude of different parameters.

The central quantity is the Rayleighian $R=W+\dot{F}$, a functional of $Q_{\alpha \beta}$ (nematic order parameter), $u_{\alpha}$ (displacement from equilibrium) and their derivatives. In this version of the variational principle, $W$ represents the dynamic energy dissipation function and $\dot{F}$ represents the rate of change of the free energy [4,11]. The dynamical equations for $Q_{\alpha \beta}$ and $u_{\alpha}$ are obtained by minimizing $R$ with respect to $\dot{Q}_{\alpha \beta}$ and $\dot{u}_{\alpha}$ with $Q_{\alpha \beta}$ and $u_{\alpha}$ held fixed. The dissipation function $W$ is defined by [9]

$$
W=\int \mathrm{d}^{3} r\left[\frac{\gamma}{2} \dot{Q}_{\alpha \beta} \dot{Q}_{\alpha \beta}+\frac{\tilde{\eta}_{\mathrm{op}}}{2} \dot{u}_{\alpha \beta} \dot{u}_{\alpha \beta}+\epsilon \dot{Q}_{\alpha \beta} \dot{u}_{\alpha \beta}\right],
$$

with the deformation $u_{\alpha \beta}=\frac{1}{2}\left(\frac{\partial u_{\alpha}}{\partial x_{\beta}}+\frac{\partial u_{\beta}}{\partial x_{\alpha}}\right)$. The coefficients in (1) are generalized viscosities. The $\epsilon$-term describes the dissipation due to the microscopic dynamical coupling between the nematic order parameter and deformation. In equation (1), we have corrected an oversimplification from an earlier paper [4]. In fact, the coefficient of the $\dot{u}_{\alpha \beta} \dot{u}_{\alpha \beta}$-term should have appeared as an operator $\tilde{\eta}_{\mathrm{op}}$ which Fourier transforms as the frequency dependent viscosity [12]: $\eta(\omega)=\eta_{0} /\left(1+i \omega \tau_{\mathbf{R}}\right)$, where $\tau_{\mathrm{R}} \sim 10^{-6}-10^{-7} \mathrm{~s}$ is the Rouse time for the polymer chains between crosslinks.

The free energy is given by [6]

$$
F-F_{0}=\int \mathrm{d}^{3} r\left[\frac{A(T)}{2} Q_{\alpha \beta} Q_{\alpha \beta}-U Q_{\alpha \beta} u_{\alpha \beta}+\frac{\mu_{0}}{2} u_{\alpha \beta} u_{\alpha \beta}-\sigma_{\alpha \beta}^{\mathrm{app}} u_{\alpha \beta}\right],
$$

where $A(T)=a\left(T-T^{\star}\right)$ defines the cooling transition temperature for the virtual second order phase transition and $\mu_{0} / 2$ is the second Lamé coefficient of the gel (considered as incompressible 
but deformable). This form of the free energy is valid near the transition temperature where $Q_{\alpha \beta}$ and $u_{\alpha \beta}$ are small. Further discussion on this point is postponed to the Conclusion. The actual first order transition happens at $T=T_{\mathrm{NI}}$ which is slightly higher than $T^{\star}\left(T_{\mathrm{NI}}-T^{\star}\right.$ lies around $1 \mathrm{~K})$ [13]. Due to the presence of spontaneous elongation in the ordered phase, transition temperatures $\left(T^{\star}\right.$ and $\left.T_{\mathrm{NI}}\right)$ will be renormalized to $T^{\star}+U^{2} /\left(a \mu_{0}\right)$ and $T_{\mathrm{NI}}+U^{2} /\left(a \mu_{0}\right)=T_{\mathrm{NI}}^{\star}[6]$. One can estimate this shift of the transition temperatures $U^{2} /\left(a \mu_{0}\right)$ : it is of the order of a few kelvins in the case where nematogens are in the main chain and millikelvins for nematogens in the side chains [14]. The time derivative of $F$ is [4]

$$
\dot{F}=\int \mathrm{d}^{3} r\left[\Phi_{\alpha \beta} \dot{Q}_{\alpha \beta}+K_{\alpha \beta} \dot{u}_{\alpha \beta}\right]
$$

where

$$
\begin{aligned}
\Phi_{\alpha \beta} & =A(T) Q_{\alpha \beta}-U u_{\alpha \beta} \\
K_{\alpha \beta} & =\mu_{0} u_{\alpha \beta}-U Q_{\alpha \beta}-\sigma_{\alpha \beta}^{\text {app }}
\end{aligned}
$$

The quantities $-\Phi_{\alpha \beta}$ and $-K_{\alpha \beta}$ are generalized forces in the de Groot sense [15]. The phenomenological dynamical equations are given by [4]

$$
\begin{aligned}
\dot{Q}_{\alpha \beta} & =-\frac{1}{\gamma}\left[A(T) Q_{\alpha \beta}{ }^{\prime}-U u_{\alpha \beta}+\epsilon \dot{u}_{\alpha \beta}\right] \\
\rho \frac{\partial \dot{u}_{\alpha}}{\partial t} & =\frac{\partial \sigma_{\alpha \beta}}{\partial x_{\beta}}
\end{aligned}
$$

where the stress tensor is

$$
\sigma_{\alpha \beta}=\mu_{0} u_{\alpha \beta}-U Q_{\alpha \beta}+\frac{\eta_{0}}{\tau_{\mathrm{R}}} \int_{-\infty}^{t} \mathrm{~d} t^{\prime} \mathrm{e}^{-\left(t-t^{\prime}\right) / \tau_{\mathrm{R}}} \dot{u}_{\alpha \beta}\left(t^{\prime}\right)+\epsilon \dot{Q}_{\alpha \beta}-\sigma_{\alpha \beta}^{\mathrm{app}} .
$$

Similar dynamical equations have been derived using the de Groot [15] phenomenological approach [16].

The order of magnitude of parameters in $(1,2)$ can be estimated $[6,17]$ using the fraction of crosslinked monomers ( $\nu \ll 1$ for weakly crosslinked gels), the pure nematic virtual second order transition temperature $\left(T^{\star}\right)$, the volume per monomer $(V)$, the nematic relaxation time $\left(\tau_{\mathrm{N}} \sim 10^{-5}-10^{-7} \mathrm{~s}[13]\right)$ and the gel relaxation time $\left(\tau_{\mathrm{g}} \simeq \tau_{\mathrm{R}}\right)$

$$
\begin{aligned}
A(T) & =a\left(T-T^{\star}\right) \simeq \frac{k_{\mathrm{B}}\left(T-T^{\star}\right)}{V} \sim \frac{k_{\mathrm{B}} T}{V} \\
\mu_{0} & \sim U \sim \frac{k_{\mathrm{B}} T \nu}{V} \\
\gamma & \sim \frac{k_{\mathrm{B}} T \tau_{\mathrm{N}}}{V} \\
\eta_{0} & \sim \frac{k_{\mathrm{B}} T \tau_{\mathrm{g}} \nu}{V}
\end{aligned}
$$

We have deliberately replaced $\tilde{\eta}_{\mathrm{op}}$ by its characteristic value $\eta_{0}$, and will continue to do so for the rest of this paper. This does not affect the conclusions of the thermodynamic calculations presented here.

In a similar manner, three characteristic relaxation times can be defined from various combinations of parameters of $(1,3)$ [4]. First, $\tau_{\mathrm{N}}=\gamma / A(T) \sim 10^{-5}-10^{-7} \mathrm{~s}$ is the nematic relaxation 
a)

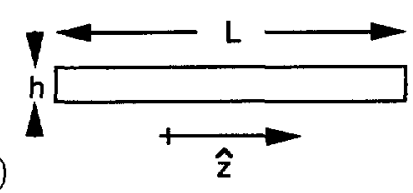

b)

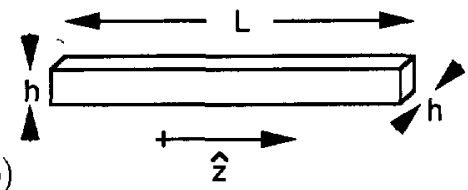

Fig. 1. - Slab and fiber geometries. In both of them, $h$ is assumed to be very small compared to $L$.

time [13]. In (9), it was assumed that the generalized viscosity $\gamma$ has a weak dependence on temperature. The important physical feature is that the ratio $\gamma / A(T)$ be, to a good approximation, equal to $\tau_{\mathrm{N}}$ sufficiently far from the transition. Second, $\tau_{\mathrm{g}}=\eta_{\mathrm{a}} / \mu_{0} \simeq \tau_{\mathrm{R}}$ is the gel relaxation time. This is very sensitive to the defects in the structure (dangling ends or uncrosslinked chains). In the present paper, we assume a rather rapid $\tau_{\mathrm{R}} \sim 10^{-6} \mathrm{~s}$. The last relaxation time $\tau_{\text {micro }}=-\epsilon / U$ comes from the microscopic coupling between nematogens and the polymer network. Its physical meaning can be seen by setting $\sigma_{\alpha \beta}$ and $u_{\alpha \beta}$ to zero in (8): the resulting expression describes the relaxation of the order parameter due to the presence of the polymer chains. It is supposed here to be very fast and we take $\tau_{\text {micro }} \leq \tau_{\mathrm{N}} \simeq \tau_{\mathrm{R}}$. In the present calculation, we consider only the dominant dissipative processes: polymer-polymer dissipation $\left(\dot{u}_{\alpha \beta} \dot{u}_{\alpha \beta}\right.$-term) and nematic-nematic dissipation $\left(\dot{Q}_{\alpha \beta} \dot{Q}_{\alpha \beta}\right.$-term). Thus, we neglect the $\epsilon$-term in (1).

The 2D slab geometry was studied (Fig. 1a) in relation to the fiber geometry of muscles (Fig. 1b). In this case, the dynamical equations derived from $R$ reduce to (for $u_{z z} \propto Q_{z z} \propto$ $\left.e^{\imath \omega_{k} t}\right)$

$$
-\rho \omega_{k}^{2} u_{z z}=\mu\left(\omega_{k}\right) \frac{\partial^{2} u_{z z}}{\partial z^{2}}
$$

with

$$
\mu\left(\omega_{k}\right)=4\left(\mu_{0}+\frac{i \omega_{k} \eta_{0}}{1+i \omega_{k} \tau_{\mathrm{R}}}-\frac{U^{2}}{A(T)} \frac{1}{1+i \omega_{k} \tau_{\mathrm{N}}}\right) .
$$

The relaxation times for the nematic order parameter $\left(\tau_{\mathrm{N}}\right)$ and for the deformation $\left(\tau_{\mathrm{R}}\right)$ are close to one another. Considering the dynamics in such a case can be very difficult (to be convinced, see $(6,7,10,11))$. Two illustrative limiting cases represent good starting points for the comprehension of dynamics in this system. First, suppose that the relaxation for $Q_{\alpha \beta}$ is much smaller than that of $u_{\alpha \beta}$. This is the condition of spontaneous relaxation of nematic constraints $\left(\tau_{\mathrm{N}} \ll \tau_{\mathrm{R}}\right)$. The dynamics of the dry nematic gel will be governed by the slowest variable $\left(u_{\alpha \beta}\right) ; Q_{\alpha \beta}$ being fast enough to instantaneously adjust to minimize locally the free energy $F$ (Eq. (2)) for a given $u_{\alpha \beta}$. Mathematically, this condition is $\delta F / \delta Q_{\alpha \beta}=0$ which yields the minimum value of $Q_{\alpha \beta}$, namely

$$
Q_{\alpha \beta}^{\star}=\frac{U}{A(T)} u_{\alpha \beta}
$$

This relation can be used at the transition temperature $T_{\mathrm{NI}}$ and at higher temperatures, where $A(T)$ is small but positive, to prevent pathological behaviors. Second, if the relaxation time for $u_{\alpha \beta}$ is much smaller than that of $Q_{\alpha \beta}$, we are in the condition of spontaneous relaxation of mechanical constraints $\left(\tau_{\mathrm{R}} \ll \tau_{\mathrm{N}}\right)$. The dynamics will be that of the nematic order parameter $Q_{\alpha \beta}$, since $u_{\alpha \beta}$ is fast to relax to its equilibrium value $u_{\alpha \beta}^{\star}$ given by $\delta F / \delta u_{\alpha \beta}=0$

$$
u_{\alpha \beta}^{\star}=\frac{U}{\mu_{0}} Q_{\alpha \beta} .
$$


a)

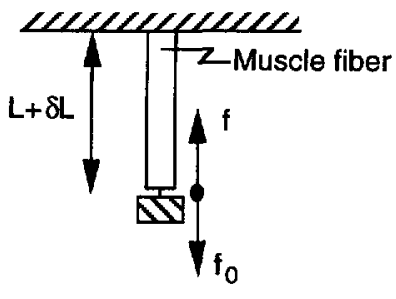

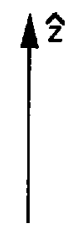

b)

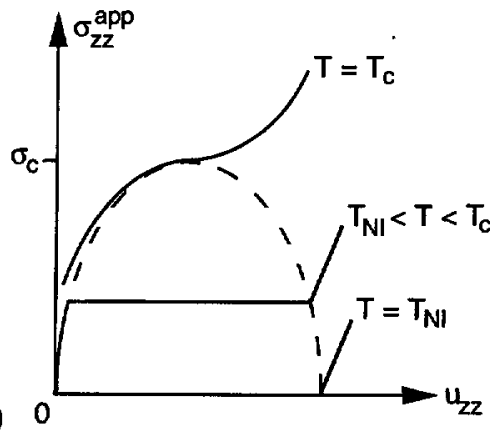

c)

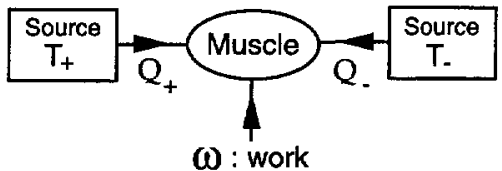

$\omega$ : work

Fig. 2. - a) Schematic set-up for an isotonic experiment using an artificial muscle fiber. b) Stressstrain relation in nematic gels showing a few isotherms (according to [6]). Under the dashed, we find the metastable region of the phase transition. c) Associated heat machine defining heat flow and work.

In each cases, corresponding conditions (12) or (13) can then be replaced in $F$ (Eq. (2), see [6]) or in generalized forces $(4,5)$.

In both of these cases $\left(\omega_{k} \tau_{\mathrm{R}}\right.$ finite with $\omega_{k} \tau_{\mathrm{N}}=0$ and $\omega_{k} \tau_{\mathbf{R}}=0$ with $\omega_{k} \tau_{\mathrm{N}}$ finite $), \mu\left(\omega_{k}\right)$ becomes

$$
\mu\left(\omega_{k}\right)=\tilde{\mu}_{0}+\left(\tilde{\mu}_{\infty}-\tilde{\mu}_{0}\right) \frac{i \omega_{k} \tau}{1+i \omega_{k} \tau}
$$

A dimensionless parameter $r=\left(c_{0} \tau\right) / h$ is used to identify the relaxation regime. For $r<1$, the system is in the fast relaxation regime and, for $r>1$ it is in the slow relaxation regime. The propagation of a step-like deformation or nematic ordering front has been studied for both of these regimes. The conclusions are that this front propagates at a speed $c_{0}=2 c_{\mathrm{T}}$ ( $c_{\mathrm{T}}=\sqrt{\tilde{\mu}_{0} / 2 \rho}$ is the transverse speed of sound), and has a width $\delta$ which grows as the square root of time $\left(\delta=c_{0} \sqrt{t \tau}\right)$. The associated shape relaxation time is $\tau_{\text {shape }}=L /\left(4 c_{\mathrm{T}}\right)$, in the range of milliseconds for a sample of size $L=10 \mathrm{~cm}$. This is very fast when compared to typical time scales of swelling experiments. However, the time for the contraction/elongation cycle is not $\tau_{\text {shape }}$ it is regulated by heat diffusion in the nematic gel which is of the order of seconds.

\section{Irreversible Thermodynamics}

This Section is devoted to irreversible thermodynamics of artificial muscles in general, and particular energetic considerations of nemato-mechanical conversion. We will be especially interested by the irreversible degradation of entropy governed by the dissipation function $W$ (Eq. (1)), during an operating cycle.

3.1. Operating Contraction/Elongation Cycle. - Consider the set-up of Figure 2a where an artificial muscle fiber of section $h^{2}$ is attached to a weight of mass $m=f_{0} / g$ (applied stress $\left.\sigma_{0}=f_{0} / h^{2}\right)$. The length of the fiber is $L$ in the undeformed state, $L+\delta L$ during 


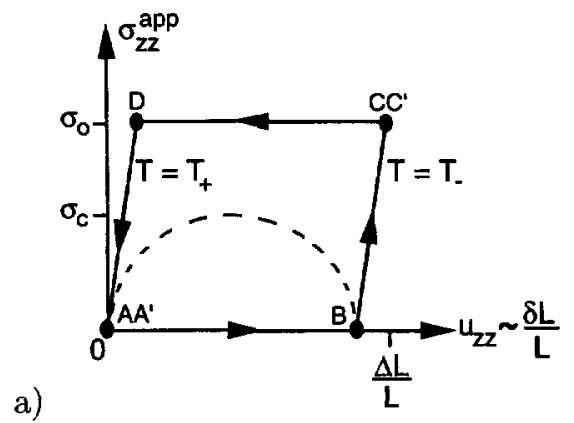

b)

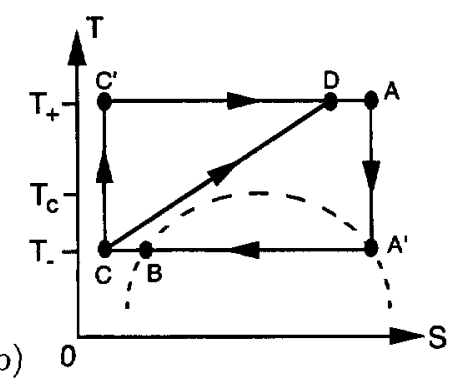

Fig. 3. - Operating cycle described in the text. a) The stress-strain $\left(\sigma_{z z}^{\text {app }}\right.$ versus $\left.u_{z z}\right)$ diagram. The dashed line is the coexistence curve. b) The temperature-entropy ( $T$ versus $S$ ) diagram. The exact location of the segment $\mathrm{C} \rightarrow \mathrm{D}$ depends on the ratio $\tau_{\text {thermal }} / \tau_{\text {shape }}$. We can see the existence of a mechanical critical point at $\left(T=T_{\mathrm{c}}\right.$ and $\left.\sigma_{z z}^{\mathrm{app}}=\sigma_{\mathrm{c}}\right)$.

the cycle and $L+\Delta L$ at full extension. The $\sigma_{z z}^{\text {app }}$ versus $u_{z z}$ diagram of a nematic gel is given in Figure $2 \mathrm{~b}$ (taken from [6]) and the corresponding heat machine is schematically represented in Figure $2 c$ to define notations for heat sources and work [18]. It will be assumed that the characteristic time scale for contraction and elongation is $\tau_{\text {shape }} \simeq 10^{-3} \mathrm{~s}$, since this is the realistic dynamic time scale of actual muscles [1]. Clever experimental set-ups or material designs are [8], and should be, studied to bring the contraction/elongation cycle of dry nematic gels to this milleseconds dynamic range. Thus, we will assume the characteristic time for thermal conduction through the sample $\left(\tau_{\text {thermal }}\right)$ to be either shorter or of the order of $\tau_{\text {shape }}$. This will modify slightly the cycle if $\tau_{\text {thermal }} \simeq \tau_{\text {shape }}$ or $\tau_{\text {thermal }} \ll \tau_{\text {shape }}$.

The cycle described below is constructed by reasoning on free energy (2) and entropy only (non-dissipative cycle). It is used to identify the conditions under which work and, thus, dissipation (see Eq. (1)) are present in the actual dissipative and irreversible cycle.

The schematic operating cycle for the nematic gel based artificial muscles, to be technologically applicable and reliable, must be very simple. Considering this and the shape of the stress-strain diagram for our system (Fig. 2b and [6]), we opt for a cycle composed of two isotonic $\left(\sigma_{z z}^{\text {app }}=\right.$ cte $)$ and two isothermal $(T=$ cte $)$ segments.

We start at point $\mathrm{A}$ in the $\sigma_{z z}^{\mathrm{app}}$ versus $u_{z z}$ diagram (Fig. 3a) and $T$ versus $S$ diagram (Fig. $3 \mathrm{~b})$ where the sample is unstrained $\left(\sigma_{z z}^{\text {app }}=0\right)$, undeformed $\left(u_{z z}=0\right)$ and at temperature $T=T_{+}>T_{\mathrm{c}} . T_{\mathrm{c}}$ is the critical temperature above which the distinction between nematic and isotropic phases is no longer relevant [6]. The difference $T_{\mathrm{c}}-T^{\star}$ can be calculated to be of the order of $T_{\mathrm{NI}}-T^{\star}$ for our system. Typically, $T_{\mathrm{c}}=T^{\star}+b\left(T_{\mathrm{NI}}-T^{\star}\right)$ where $b$ can be estimated to be around 2 [14]: i.e. $T_{\mathrm{c}}-T^{\star} \simeq 2 \mathrm{~K}$. The sample is cooled reversibly to $T=T_{\mathrm{NI}}$ (segment $\mathrm{A} \rightarrow \mathrm{A}^{\prime}$ ) without deformation and no work is performed. The entropy does not change during the process (Fig. 3b) since it depends on $u_{z z}$ and not directly on $T$ (at least for mean field models). The temperature is reduced even further to $T_{-}$just below $T_{\mathrm{NI}}$ (segment $\mathrm{A}^{\prime} \rightarrow \mathrm{B}$ ). The system elongates and exchanges a quantity of heat $\mathcal{Q}_{-}$with the heat bath at $T=T_{-}$. This transformation is reversible for $\tau_{\text {thermal }} \simeq \tau_{\text {shape }}$ and irreversible for $\tau_{\text {thermal }} \ll \tau_{\text {shape }}$ (even for a non-dissipative cycle). The fact that segment $\mathrm{A}^{\prime} \rightarrow \mathrm{B}$ is performed at $\sigma_{z z}^{\mathrm{app}}=0$ and across the metastable region insures us that it is an isotherm for any $\tau_{\text {thermal }}$. The elongation stops at point $\mathrm{B}$. In the segment $\mathrm{B} \rightarrow \mathrm{C}$, a load $\sigma_{0}$ is applied slowly at constant temperature $T_{-}$to achieve a final deformation $\Delta L / L$. The excess elongation in going from $\mathrm{B} \rightarrow \mathrm{C}$ is small compared to the elongation in going from $\mathrm{A}^{\prime} \rightarrow \mathrm{B}$ and thus the entropy does not change much 
(see Fig. 3b). Note also that, in this particular case, the stress is applied in the direction of the optical axis $(\hat{\mathbf{z}})$ : no director reorientation should occur. Thus, the nematic gel should not experience soft elasticity [10] or polydomain structure [5], and is expected to exhibit elastic properties characteristic of normal rubbers.

The contraction process (segment $\mathrm{C} \rightarrow \mathrm{D}$ in Fig. 3a) depends on $\tau_{\text {thermal }}$. Two limiting cases are interesting: $\tau_{\text {thermal }} \simeq \tau_{\text {shape }}$ and $\tau_{\text {thermal }} \ll \tau_{\text {shape }}$. For $\tau_{\text {thermal }} \simeq \tau_{\text {shape }}$, the sample is heated by exchanging a quantity of heat $\mathcal{Q}_{+}$with a source at $T=T_{+}$. This can be achieved depositing heat by a laser; the light pulse duration being $\tau_{\text {thermal }}$ and $\mathcal{Q}_{+}$calculated to bring the sample from $T=T_{-}$to $T=T_{+}$[8]. During heating the sample realizes the equilibrium deformation instantaneously: the transformation is quasi-static and the system goes directly from $\mathrm{C} \rightarrow \mathrm{D}$ (Fig. $3 \mathrm{~b}$ ). However, if $\tau_{\text {thermal }} \ll \tau_{\text {shape }}$ (with high intensity lasers, for example), the scenario is different. In a first stage $\left(\mathrm{C} \rightarrow \mathrm{C}^{\prime}\right)$, the system is brought to temperature $T_{+}$by fast deposition of heat $\mathcal{Q}_{+}$without deformation $\left(\tau_{\text {thermal }} \ll \tau_{\text {shape }}\right)$ and no work is performed. The second stage is an isothermal contraction $\left(\mathrm{C}^{\prime} \rightarrow \mathrm{D}\right)$ converting part of the heat $\mathcal{Q}_{+}$into useful work. In this case, the system is out of equilibrium and thus the transformation is irreversible (even for a non-dissipative cycle). In either case, the $\sigma_{z z}^{\text {app }}$ versus $u_{z z}$ diagram (Fig. 3a) is unaltered. In the last segment $(\mathrm{D} \rightarrow \mathrm{A})$, the stress $\sigma_{0}$ is taken off and the system goes back to the undeformed state along an isotherm $\left(T=T_{+}\right)$. During this process, the change in $u_{z z}$ (and, thus, the change in S) is small (Figs. 3a and 3b).

The motive segments of the cycle can be identified: elongation $\left(\mathrm{A}^{\prime} \rightarrow \mathrm{B}\right)$ and contraction $\left(\mathrm{C} \rightarrow \mathrm{D}\right.$ or $\mathrm{C}^{\prime} \rightarrow \mathrm{D}$ depending on $\left.\tau_{\text {thermal }}\right)$. During these transformations, a deformation front, of width $\delta$, travels through the sample at speed $c_{0}[4]$ under a given load.

3.2. Estimation of WORK fOR the Operating CyCle. - We now turn to the work performed by the nematic gel during the cycle presented above (Sect. 3.1). The evaluation of work from equation (2) using the full form of $Q_{\alpha \beta}(\mathbf{r}, t)$ and $u_{\alpha \beta}(\mathbf{r}, t)$ [4] is mathematically very difficult. We choose to calculate work for the quasi-static cycle $\left(\tau_{\text {thermal }} \simeq \tau_{\text {shape }}\right)$ because it gives insight on the important parameters for the process, especially size dependence. The other limit $\left(\tau_{\text {thermal }} \ll \tau_{\text {shape }}\right)$ can be shown to give the same result since the $\sigma_{z z}^{\text {app }}$ versus $u_{z z}$ diagram is unaltered by the value of $\tau_{\text {thermal }}$. The operating temperatures $T_{+}$and $T_{-}$are taken to be close to the transition region while respecting $T_{+}>T_{\mathrm{c}}$ and $T_{-}<T_{\mathrm{NI}}$. The difference $T_{+}-T_{-}$is small so that we can neglect the heat capacity of the system in the energy balance. The work $\mathcal{W}$ is given by the area of cycle $\mathrm{AA}^{\prime} \mathrm{BCD}$ of Figure $3 \mathrm{a}$

$$
\mathcal{W}=-\int \mathrm{d}^{3} r \oint \sigma_{z z}^{\mathrm{app}} \mathrm{d} u_{z z} \simeq \sigma_{0} \int \mathrm{d}^{3} r \int_{\mathrm{C}}^{\mathrm{D}} \mathrm{d} u_{z z} .
$$

Note that with definitions of Figure 2c, $\mathcal{W}<0$ means that the system produces work.

To get typical orders of magnitude, it is much easier to use a number of approximations, keeping the essential features of our phenomenological calculation. It is supposed that mechanical constraints are instantaneously relaxed (see Sect. 2), giving

$$
\mathrm{d} u_{z z}=\dot{u}_{z z} \mathrm{~d} t=\frac{U}{\mu_{0}} \dot{Q}_{\alpha \beta} \mathrm{d} t
$$

It can be shown by a similar calculation that all the results presented here are also valid for the condition of spontaneous relaxation of nematic constraints. The main approximation is to replace

$$
\dot{Q}_{z z}=\frac{\mathrm{d} Q_{z z}}{\mathrm{~d} t} \sim \pm \frac{Q^{\star}}{\Delta t}
$$


where $\Delta t=\delta / c_{0}=\sqrt{t \tau}$ is the typical time taken by the deformation front to cross a certain point and $Q^{\star} \simeq 0.44$ is the value of $Q_{\alpha \beta}$ just below $T_{\mathrm{NI}}$. This is a rough first order approximation of the time derivative of $Q_{\alpha \beta}$. The plus sign (+) in (17) is taken for elongation (segment $\mathrm{A}^{\prime} \rightarrow \mathrm{B}$ in Fig. 2b), while the minus sign (-) is for contraction (segment $\mathrm{C} \rightarrow \mathrm{D}$ ).

With this crude approximation, we can write

$$
\mathcal{W} \simeq \sigma_{0} \frac{U}{\mu_{0}} \int \mathrm{d}^{3} r \int_{\mathrm{C}}^{\mathrm{D}} \dot{Q}_{z z} \mathrm{~d} t \simeq-\sigma_{0} h^{2} L \frac{U}{\mu_{0}} Q^{\star} \int_{\mathrm{C}}^{\mathrm{D}} \frac{\mathrm{d} t}{\sqrt{t \tau}} .
$$

The limits of integration in (18) are the initial. $\left(t_{\mathrm{i}}\right)$ and final $\left(t_{\mathrm{f}}\right)$ times for elongation and contraction. $t_{\mathrm{i}}$ is the time taken by the front to have a width equal to half the thickness of the fiber: $t_{\mathrm{i}}=h /\left(2 c_{0} r\right)$. This is equivalent to a short wavelength cut-off for our continuum approach. $t_{\mathrm{f}}$ is the time taken by the front to go across half of sample: $t_{\mathrm{f}}=L /\left(2 c_{0}\right)$. There are two fronts coming from both ends of the sample. The work is given by

$$
\mathcal{W} \simeq-2 \sigma_{0} h^{2} L \frac{U}{\mu_{0}} Q^{\star} \int_{h /\left(2 c_{0} r\right)}^{L /\left(2 c_{0}\right)} \frac{\mathrm{d} t}{\sqrt{t \tau}}=-2 \sqrt{2} \sigma_{0} h^{2} L Q^{\star} \frac{U}{\mu_{0}}\left(\frac{1}{\sqrt{x r}}-\frac{1}{r}\right),
$$

where $x=h / L \ll 1$ (by assumption, see Fig. 1) and requires that $r>x$ to have a system that produces work $(\mathcal{W}<0)$. This limits the value of $x=h / L \ll 1$ in the $r<1$ regime, but not in the $r>1$ regime. We can use the condition of spontaneous relaxation of mechanical constraints (13) and apply it at the beginning of contraction to yield

$$
u_{z z \max }=\frac{\Delta L}{L}=\frac{U}{\mu_{0}} Q^{\star},
$$

where $L+\Delta L$ is the length of the fiber at full elongation

$$
\mathcal{W} \simeq 2 \sqrt{2} \sigma_{0} h^{2} \Delta L\left(\frac{1}{r}-\frac{1}{\sqrt{x r}}\right) .
$$

Finally, noting that the elastic energy stored $\sigma_{0} \Delta L / L$ will be roughly equal to the typical thermal energy injected $a\left(T_{+}-T_{-}\right)\left(Q^{\star}\right)^{2}$ (see Eq. (2))

$$
\mathcal{W} \sim 2 \sqrt{2} a\left(T_{+}-T_{-}\right)\left(Q^{\star}\right)^{2} h^{2} L\left(\frac{1}{r}-\frac{1}{\sqrt{x r}}\right) .
$$

Since $r>x, \mathcal{W}<0$ and the muscle produces work as long as $T_{+}>T_{-}$. This is right only for small temperature differences $T_{+}-T_{-}$, in which case the heat capacity of the system can be neglected.

3.3. Estimation of Losses for the Operating Cycle and Muscular Efficiency. The losses in an operating cycle are calculated by integrating the dissipation function $W$ over the whole cycle. Note first that the parameters of the dissipation function are supposed to have no strong dependence on temperature. For our cycle (Sect. 3.1), this means that total losses $\mathcal{P}$ are twice those occurring during the contraction, for example,

$$
\mathcal{P}=\oint W \mathrm{~d} t \simeq 2 \int_{\mathrm{C}}^{\mathrm{D}} W \mathrm{~d} t
$$

Using the previous approximations (Sect. 3.2), $W$ becomes

$$
W \simeq \frac{3 h^{2} L\left(Q^{\star}\right)^{2}}{4}\left(\gamma+\frac{\eta_{0} U^{2}}{\mu_{0}^{2}}\right) \frac{1}{(\Delta t)^{2}}
$$


and

$$
\mathcal{P} \simeq \frac{3 h^{2} L\left(Q^{\star}\right)^{2}}{2 \tau}\left(\gamma+\frac{\eta_{0} U^{2}}{\mu_{0}^{2}}\right) \ln \left(\frac{r}{x}\right) \simeq \frac{3}{2}\left(a T_{\mathrm{NI}}\right) h^{2} L\left(Q^{\star}\right)^{2} \ln \left(\frac{r}{x}\right)
$$

since $\eta_{0} U^{2} / \mu_{0}^{2} \ll \gamma$ for $\nu \rightarrow 0$ (weakly crosslinked gel, see Sect. 2) and that $\gamma / \tau \simeq\left(a T_{\mathrm{NI}}\right)$ typically (see Eq. (9)). Recalling that in order to have production of work $r$ must be greater than $x$ (Sect. 3.2): it insures us that $\mathcal{P}>0$. Note that $r / x=L /\left(c_{0} \tau\right)$ defines a new characteristic parameter for the system and the losses per unit volume are a function of $\ln \left[L /\left(c_{0} \tau\right)\right]$.

The muscular efficiency can then be approximately given by the ratio of recuperated work over losses during the operating cycle, and using $(22,25)$,

$$
\eta_{\text {muscular }} \simeq \frac{-\mathcal{W}}{\mathcal{P}} \simeq \frac{4 \sqrt{2}}{3}\left(\frac{T_{+}-T_{-}}{T_{\mathrm{NI}}}\right) \frac{\frac{1}{\sqrt{x r}}-\frac{1}{r}}{\ln \left(\frac{r}{x}\right)}
$$

Numerical values can be replaced in (26) to estimate the theoretical muscular efficiency of the nematic gel. Taking $c_{0} \tau=0.3 \mathrm{~mm}, L=10 \mathrm{~cm}$ and $\left(T_{+}-T_{-}\right) / T_{N I}=10^{-2}, \eta_{\text {muscular }}$ will depend on the relaxation regime $(r<1$ or $r>1)$. For $r=0.1$, which imposes that $h=3 \mathrm{~mm}$, we find $\eta_{\text {muscular }} \simeq 13 \%$. For $r=10(h=0.03 \mathrm{~mm}), \eta_{\text {muscular }} \simeq 3.3 \%$. This is of the order of the efficiency of actual muscles [1].

\section{Conclusions}

In this article, we have reviewed the dynamical properties of dry nematic gels using a variational approach based on continuum phenomenological free energy and dissipation function coupling the nematic order parameter and the deformation of the media. The irreversible thermodynamical properties of the contraction/elongation cycle were discussed. We have shown, using approximations, that the muscular efficiency of nematic gel based artificial muscle is comparable to that of real muscles. However, a number of remarks are in order.

- Since the transition is first order, the quantity of heat $\mathcal{Q}_{+}$and $\mathcal{Q}_{-}$(Fig. 2c) will be roughly proportional to the latent heats of transition. In all practical cases this latent heat of transition is enormous when compared to work that can be performed. Finally, the thermodynamical efficiency |work/latent heat| will be very small [8].

- One can use scaling arguments to justify the size dependence of $\eta_{\text {muscular }}$. Take the case of a real muscle in the fiber geometry (Fig. 1b). The scaling muscular efficiency $\eta_{\text {muscular }}^{\text {scaling }}$ will be

$$
\eta_{\text {muscular }}^{\text {scaling }} \sim\left|\frac{\text { work }}{\text { losses }}\right|,
$$

where work will go like $f \mathrm{~d} l$. The force $f$ will depend on the cross section of the fiber $f \sim h^{2}$. The elongation $\mathrm{d} l$ will scale like the length of the fiber $\mathrm{d} l \sim L$. The losses are characterized by generation of heat and will scale like the surface through which it must diffuse: $\mid$ losses $\mid \sim 4 h L+2 h^{2}$.

$$
\eta_{\text {muscular }}^{\text {scaling }} \sim \frac{h^{2} L}{4 h L+2 h^{2}} \sim \frac{h}{2+h / L} .
$$

If $h / L$ is small, $\eta_{\text {muscular }}^{\text {scaling }} \sim h$ to first order in $h / L$. 
To compare this result with $\eta_{\text {muscular }}$ (Eq. (26)), we will have problems dealing with $\ln (r / x)$. However, we know that $r$ must be greater than $x$ (Sect. 3.2), so we take $r=x+\Delta x$ with $\Delta x / r \ll 1$, and develop $\eta_{\text {muscular }}$

$$
\eta_{\text {muscular }} \sim \frac{\frac{1}{\sqrt{x r}}-\frac{1}{r}}{\ln (r / x)} \sim \frac{1}{r}\left(\frac{1-3 \frac{r-x}{x}}{1-\frac{r-x}{2 x}}\right) \sim h
$$

to first order in $h$. This is to be compared to (28), and thus gives a hint that, even if we made strong approximations, the $h$ scaling remains correct for small $h$. Moreover, if we vary the dimensions of the fiber at constant volume, we end up with $\eta_{\text {muscular }} \sim 1 / \sqrt{L}$ : cube shaped muscles would be most efficient.

- A clear and logical conclusion to this calculation is that the various parameters have to be optimized while respecting the system's intrinsic constraints. First, to have production of work, $r>x$ (see Sect. 3.2). This imposes limits on $h$ and $L$ with respect to $c_{0} \tau$. Now consider heat diffusion in one of the fiber's transverse direction ( $\hat{x}$ or $\hat{y}$ in Fig. 1 ), with finite thermal conductivity $\kappa$. Suppose a cooling process where a nematic ordering front has reached a distance $x(t)$ from the cold source at temperature $T$. The heat current will be approximately given by

$$
J_{\mathrm{Q}} \simeq \kappa \frac{\left(T_{\mathrm{NI}}-T\right)}{x(t)} \simeq \tilde{L} \frac{\mathrm{d} x(t)}{\mathrm{d} t}
$$

where $\tilde{L}$ is the heat of transformation. Rearranging (30) and integrating over a distance $h$, we get the approximate expression for the actual cooling time

$$
\tau_{\text {thermal }} \sim \frac{h^{2}}{D_{\text {th }}} \frac{T_{\mathrm{NI}}}{T_{\mathrm{NI}}-T}
$$

where $D_{\mathrm{th}}=\kappa T_{\mathrm{NI}} / \tilde{L}$ is the thermal diffusion constant. In view of this, if $D_{\mathrm{th}}$ cannot be optimized, one will have to choose $h$ by balancing the tendency to have $\tau_{\text {thermal }}$ small and $\eta_{\text {muscular }}$ large.

Finally, one would gain a lot by having $T_{+}-T_{-}$large, but this is not always technologically possible. Moreover, we have neglected everywhere the heat capacity of the gel: this is justifiable for small temperature difference $T_{+}-T_{-}$.

- We have used very strong approximations to calculate $\mathcal{W}$ and $\mathcal{P}$ (Eqs. $(22,25)$ ) replacing $\dot{Q}_{z z}$ by $Q^{\star} / \Delta t$, neglecting all spatial variations of $Q_{z z}(z, t)$. In this respect, we used an uncontrollable approximation (one with no small control parameter). However, we feel that the knowledge acquired on the parameters governing the process is worthwhile, and that the size dependence of various thermodynamical quantities can be motivated.

- The work presented here is based on linear dynamical equations valid only near $T_{\mathrm{NI}}$ where the nematic order parameter and deformations are small (the region where the free energy of the system is described by (2)). The quadratic form of the free energy [6] successfully describes the main features encountered in nematic gels (renormalization of $T_{\mathrm{NI}}$, mechanical critical point, etc.). However, deep in the nematic phase, deformations are no longer small and higher order terms (schematically) $u^{3}, u^{4}$, etc. have to be included (higher order terms in $Q_{\alpha \beta}$ are implied in the free energy (2) to keep $Q_{\alpha \beta}$ finite 
for $A(T)<0$ ). In such a case, the coupling term would be more complex than the one used here $\left(-U Q_{\alpha \beta} u_{\alpha \beta}\right)$ and have contributions from (schematically) $Q u^{2}, Q^{2} u$-terms, for example. A free energy similar to the one derived by Warner et al. [10] could be a good candidate to account for non-linear effects. In developing a better model for the dynamics of dry nematic gels, near $T_{\mathrm{NI}}$ or in the nematic phase, spatial variations of the order parameter would also have to be accounted for.

Another important effect is encountered in the nematic phase: soft elasticity [10] where uniaxial strain is built without resistance below a critical deformation, which corresponds to complete alignment of the nematic director with the direction of the applied stress. This effect is not expected here since the stress applied in the segment $\mathrm{B} \rightarrow \mathrm{C}$ of Figure 3 is supposed to be along the director axis.

Finally, we are still quite far from the actual implementation of a nemato-mechanical conversion scheme in artificial muscles. Technological aspects have still to be further studied (reduction of $\tau_{\text {thermal }}$, fatigue of the material, etc.), but this scheme looks like a serious candidate.

\section{Acknowledgments}

We would like to thank M. Warner and D. Boies for useful discussions. One of us (M.H.) wishes to acknowledge the support of the Natural Sciences and Engineering Research Council of Canada (NSERC).

\section{References}

[1] Stanford A.L., Foundations in Biophysics (Academic Press, New-York, 1975).

[2] Katchalsky A. and Oplatka A., Hanbook in Sensory Physiology, vol. 1 (Springer-Verlag, Berlin, 1971); Katchalsky A., Experimentia 5 (1949) 319.

[3] Tanaka T., Fillmore D., Sun S.T., Nishio I., Swislow G. and Shah A., Phys. Rev. Lett. 45 (1980) 1636.

[4] de Gennes P.-G., Hébert M. and Kant R., Macromol. Symp. 113 (1997) 39.

[5] Küpfer J. and Finkelmann H., Makromol. Chem. Rapid Commun. 12 (1991) 717.

[6] de Gennes P.-G., Polymer Liquid Crystals, A. Cifferi, W.R. Krigbaum and R.B. Meyer, Eds. (Academic Press, New York, 1982); de Gennes P.-G., C.R. Acad. Sci. Paris B281 (1975) 101.

[7] Tanaka T., Annaka M., Ilmain F., Ishii K., Kokufuta E., Suzuki A. and Tokita M., NATO ASI Series, vol. H64 (Springer-Verlag, Berlin, 1992).

[8] de Gennes P.-G., unpublished (1996).

[9] Landau L.D. and Lifshitz E.M., Statistical Mechanics (Pergamon Press, Oxford, 1980).

[10] Warner M., Bladon P. and Terentjev E., J. Phys. II France 4 (1994) 93.

[11] Doi M., J. Chem. Phys. 79 (1983) 5080; Doi M. and Onuki A., J. Phys. II France 2 (1992) 1631.

[12] Ward I.M., Mechanical properties of solid polymers (Wiley Interscience, New York, 1971).

[13] de Gennes P.-G., Mol. Cryst. Liq. Cryst. 12 (1971) 193.

[14] Schätzle J., Kaufhold W. and Finkelmann H., Makromol. Chem. 190 (1989) 3269.

[15] de Groot S.R., Thermodynamics of irreversible processes (North-Holland, 1954).

[16] Brand H.R. and Kawasaki K., J. Phys. II France 2 (1992) 1789.

[17] Doi M., Introduction to Polymer Physics (Oxford University Press, Oxford, 1995).

[18] Lhuillier C. and Rous J., Introduction à la thermodynamique (Dunod, Paris, 1992). 